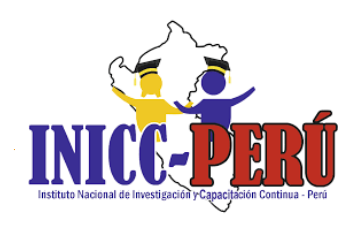

\title{
Deslinde conceptual entre educación en línea o educación a distancia
}

Conceptual demarcation between online education or distance education

\section{ENRIQUE RENTERÍA CASTRO ${ }^{1}$}

https://orcid.org/0000-0001-7028-7172

\begin{abstract}
RESUMEN: La COVID-19 logró en unos días lo que las reformas educativas desde hace 25 años no pudieron lograr: promover el uso de las herramientas digitales, en los diferentes niveles educativos, en los procesos de enseñanza y aprendizaje; así mismo transformar la organización y estructura de la escuela. Sin embargo, esta acción emergente está generando improvisaciones y descontextualización del enfoque y conceptos de la Pedagogía Virtual. Afirmar que las aplicaciones Zoom, Facebook y WhatsApp son plataformas educativas que permiten lograr exitosamente los procesos de enseñanza aprendizaje y que la educación por internet es una modalidad denominada Educación a Distancia son proposiciones erróneas que deben ser definidas y contextualizadas para no desvirtuar el enfoque científico que han logrado avanzar la Educación, la Pedagogía y la Didáctica. El propósito de este artículo pretende definir los siguientes conceptos: Educación a Distancia; Educación en Línea usando las diferentes herramientas digitales que la tecnología ha logrado desarrollar y que obligan a una definición de la Pedagogía Virtual.
\end{abstract}

Palabras clave: Educación a distancia; educación en línea; pedagogía; didáctica; currículum; plataforma educativa.

ABSTRACT: COVID-19 achieved in a few days what the educational reforms for 25 years have not been able to achieve: promoting the use of digital tools, at different educational levels, of the teaching and learning processes; likewise transform the organization and structure of the school. However, this emerging action is generating improvisations and decontextualization of the approach and concepts of Virtual Pedagogy. To affirm that the Zoom, Facebook and WhatsApp applications are educational platforms that allow the teaching-learning processes to be successfully achieved and that online education is a modality called Distance Education are erroneous propositions that must be defined and contextualized so as not to distort the scientific approach that they have managed to advance Education, Pedagogy and Didactics. The purpose of this article is to define the following concepts: Distance Education; Online Education using the different digital tools that technology has managed to develop and that require a definition of Virtual Pedagogy.

Keywords: Distance education, online education, pedagogy, didactics, curriculum, educational platform. 


\section{INTRODUCCIÓN}

\section{Antecedentes y definición de la educación a distancia}

La Educación a Distancia es una modalidad que surge durante el siglo XVIII. La definición clásica de educación a distancia se refiere a cualquier implementación formal del aprendizaje, en el que la mayor parte de la instrucción ocurre a distancia. La conjunción de los servicios impresos y el correo tradicional fincaron las bases para el desarrollo de las primeras experiencias educativas a distancia. Debemos mencionar que el primer debate referente al modelo de enseñanza, surge entre el modelo educativo de la educación patrística implementado en todo el mundo y el modelo propuesto por la primer Revolución Industrial, donde se da origen a la producción en serie y los inventos tecnológicos que promueven una nueva forma de producción y consumo, la sociedad en conjunto transforma el sistema educativo para aprovechar que los niños y jóvenes asistan a la escuela, aprendan y se capaciten para contribuir en la producción al trabajar en las fábricas.

Por tanto, la persona que tiene el interés de aprender nuevos conocimientos y poder hacer uso de las nuevas herrarmientas para la producción, se registraban en un curso denominado a distancia, el cual surge por medio del servicio postal, sistema dedicado a transportar documentos escritos, así como paquetes de pequeño o mediano tamaño, para ser trasladados alrededor del mundo, por tierra, mar o aire. A todo envío realizado a través del servicio postal se le nombro correo o correspondencia.

Cada uno de los inventos que han surgido, a lo largo de la historia, son el sustento para la generación de documentos informativos y por consecuencia la evolución de los procesos para comunicación y difusión del mensaje. El primero de estos inventos es la escritura y los primeros documentos de un sistema organizado provienen de Egipto (2400 a. C.) donde los faraones usan mensajeros para difundir sus decretos en todo el territorio, dichos decretos eran escritos con tinta en papiro y pergamino. Usado también por los babilónios, griegos y romanos. Hasta ser sustituido por el papel en el año 105 d. C.

Las instituciones de correos van abriendo en diferentes naciones; en Francia surge con Carlomago en 807; en España se tiene referencia del servicio postal en 1213; en Alemania, a mediados del XV; En México en 1580; en Inglaterra, el primer servicio postal se establece en 1635. La consolidación de este servicio tuvo también avances debido a los inventos que fueron surgiendo durante el paso de los años; entre ellos podemos señalar la imprenta del alemán Johannes Gutenberg entre los años 1449 y 1452, con la publicación del Misal de Constanza y la Biblia, respectivamente. Otro invento es el ferrocarril en el siglo XVI, en las minas de Transilvania y en Gran Bretaña en el siglo XVII; y su evolución a la locomotora de vapor en 1804.

La Educación a distancia en estos períodos se definió como una modalidad educativa, para capacitar o formar en un campo de orden práctico a aprendices o estudiantes de un oficio que se encuentran geográficamente lejos del lugar del establecimiento educativo o del domicilio del instructor; se promovió a través de documentos escritos que integran: explicación, impartición de un conocimiento o explicación de pasos para resolver una actividad específica. 
Tabla 1.

La evolución de la Educación a Distancia García Arieto (1991).

\begin{tabular}{|c|c|c|}
\hline AÑO & AUTOR & CURSO \\
\hline 1728 & $\begin{array}{c}\text { Calleb Phillips (estadoun- } \\
\text { idense) }\end{array}$ & $\begin{array}{l}\text { Cursos por correspondencia para apren- } \\
\text { der taquigrafía, se publica anuncio en el } \\
\text { periódico Boston Gazette. }\end{array}$ \\
\hline 1833 & A.J. Meuller (sueco) & $\begin{array}{l}\text { Curso de Composición por correspon- } \\
\text { dencia, sepública cambio de dirección } \\
\text { en el periódico Lunds Weckobland. }\end{array}$ \\
\hline 1840 & Issac Pitman (inglés) & $\begin{array}{l}\text { Colegio por Correspondencia sistema } \\
\text { de taquigrafía Pitman (1837) basado en } \\
\text { tarjetas. }\end{array}$ \\
\hline 1843 & $\begin{array}{l}\text { PHONOGRAPHIC CORRE- } \\
\text { SPONDENCE SOCIETY }\end{array}$ & $\begin{array}{l}\text { Institución con normatividad para for- } \\
\text { mación a distancia: recibe, corrige y } \\
\text { envía los ejercicios de taquigrafía del } \\
\text { curso por correspondencia. }\end{array}$ \\
\hline 1856 & $\begin{array}{l}\text { Charles Toussaint (francés) } \\
\text { y Gustav Langescheidt } \\
\text { (alemán) }\end{array}$ & $\begin{array}{l}\text { Instituo de idiomas por corresponden- } \\
\text { cia. }\end{array}$ \\
\hline 1858 & $\begin{array}{l}\text { Skerry College de la Univer- } \\
\text { sidad de Londres, Edimbur- } \\
\text { go, Reino Unido }\end{array}$ & $\begin{array}{l}\text { Clases a distancia para candidatos el } \\
\text { servicio civil. }\end{array}$ \\
\hline 1873 & $\begin{array}{c}\text { Ana Elit Tickner (estadoun- } \\
\text { idense) }\end{array}$ & $\begin{array}{l}\text { Asociación colectiva de escuelas por } \\
\text { correspondencia para fomentar estudios } \\
\text { en el hogar. }\end{array}$ \\
\hline 1903 & $\begin{array}{l}\text { Julio Cervera Baviera (es- } \\
\text { pañol) }\end{array}$ & $\begin{array}{l}\text { Escuela Libre de Ingenieros. Cursos } \\
\text { por correspondencia. }\end{array}$ \\
\hline 1922 & $\begin{array}{c}\text { Universidad de Pensilvania. } \\
\text { USA }\end{array}$ & $\begin{array}{l}\text { Primera institución que transmite cur- } \\
\text { sos por radio, más de } 200 \text { colegios y } \\
\text { universidades tienen licencias de radio- } \\
\text { difusión para el año } 1925 \text {. }\end{array}$ \\
\hline 1934 & $\begin{array}{c}\text { Comisión Federal de Comu- } \\
\text { nicaciones. USA }\end{array}$ & $\begin{array}{l}\text { Administra las radiofrecuencias para la } \\
\text { educación universitaria. }\end{array}$ \\
\hline 1947 & $\begin{array}{l}\text { Acción Cultural Popular Co- } \\
\text { lombia }\end{array}$ & $\begin{array}{l}\text { Modelo genuinamente latinoamericano } \\
\text { con las llamadas escuelas radiofónicas. }\end{array}$ \\
\hline 1956 & $\begin{array}{l}\text { Instituto Latinoamericano de } \\
\text { la Comunicación Educativa } \\
\text { (ILCE) }\end{array}$ & $\begin{array}{l}\text { UNESCO y México crean un organis- } \\
\text { mo regional para el mejoramiento de la } \\
\text { educación a través de la aplicación de } \\
\text { medios y recursos audiovisuales (car- } \\
\text { teles, filminas, acetatos, franelógrafo, } \\
\text { entre otros.) }\end{array}$ \\
\hline 1965 & $\begin{array}{l}\text { Universidad de Wisconsin. } \\
\text { USA }\end{array}$ & $\begin{array}{l}\text { Implementa formato de correspon- } \\
\text { dencia telefónica para capacitación de } \\
\text { médicos. }\end{array}$ \\
\hline
\end{tabular}




\begin{tabular}{|c|c|c|}
\hline 1968 & $\begin{array}{l}\text { Telesecundaria. México. La } \\
\text { idea de "teleescoula" surge } \\
\text { en Italia, de } 1958 \text { a } 1966 .\end{array}$ & $\begin{array}{l}\text { Modelo de educación mexicano creado } \\
\text { con el propósito de impartir la educa- } \\
\text { ción secundaria a través de transmisio- } \\
\text { nes televisivas, en las zonas rurales o } \\
\text { de difícil acceso de la República Mexi- } \\
\text { cana y para abatir el analfabetismo. }\end{array}$ \\
\hline 1962 & $\begin{array}{l}\text { Advanced Research Projects } \\
\text { Agency (DARPA) y AR- } \\
\text { PANET Estados Unidos. }\end{array}$ & $\begin{array}{l}\text { Red global de computadoras habilitadas } \\
\text { para intercambiar información entre } \\
\text { instituciones. Posteriormente J.C.R. } \\
\text { Licklider crea la red exclusivamente } \\
\text { militar, para tener acceso a la informa- } \\
\text { ción militar desde cualquier punto del } \\
\text { país (1969). }\end{array}$ \\
\hline 1969 & $\begin{array}{l}\text { Open University of United } \\
\text { Kingdom }\end{array}$ & $\begin{array}{l}\text { Primer modelo representativo que ofre- } \\
\text { ce estudios universitarios abiertos, es } \\
\text { decir flexibilidad de tiempo y lugar. }\end{array}$ \\
\hline 1972 & $\begin{array}{l}\text { InterNetworking Working } \\
\text { Group. Estados Unidos y } \\
\text { Noruega. }\end{array}$ & $\begin{array}{l}\text { Organización encargada de administrar } \\
\text { Internet. }\end{array}$ \\
\hline 1976 & $\begin{array}{l}\text { Coastline Community Col- } \\
\text { lege. Estados Unidos. }\end{array}$ & $\begin{array}{l}\text { Primer campus virtual integra: teléfono, } \\
\text { televisión, radio, discos, cintas para } \\
\text { cursos. }\end{array}$ \\
\hline 1979 & $\begin{array}{l}\text { NewsGroups. Estados Uni- } \\
\text { dos }\end{array}$ & $\begin{array}{l}\text { Creación de foros de discusión por es- } \\
\text { tudiantes. }\end{array}$ \\
\hline 1981 & TCP/IP & $\begin{array}{l}\text { Definición del protocolo y concepto de } \\
\text { Internet. }\end{array}$ \\
\hline 1991 & World Wide Web & $\begin{array}{l}\text { Uso de hipervínculos fórmula que per- } \\
\text { mite vincular información en forma } \\
\text { lógica y a través de las redes. }\end{array}$ \\
\hline 1997 & Web Course Tools & $\begin{array}{l}\text { Primer LMS para uso genérico en las } \\
\text { instituciones educativas }\end{array}$ \\
\hline
\end{tabular}

Igualmente, para responder mejor a las exigencias de la orientación y guía del alumno se fueron introduciendo paulatinamente en estos cursos de sólo "correspondencia", los aportes de las nacientes tecnologías audiovisuales. Durante 1830 se inicio la comunicación en la distancia a través del telégrafo y sus códigos Morse (1820). En 1876 el escocés A. Graham Bell inventó el teléfono que permitió la comunicación síncrona de forma verbal a distancia. En 1894 el joven italiano G. Marconi, inventa la radio y en 1901 se realiza la primera comunicación trasatlántica por radio, posteriormente en 1920 se abre la primera emisora de radio en Norteamérica. El teletipo de 1910 permitía el envío de mensajes escritos a distancia utilizando determinados códigos y en 1923 nace con Vladimir Zworykin, la televisión que, a partir de 1935 efectúa sus primeras emisiones regulares (García Areito, 1987).

Los cursos por televisión han sido los de mayor duración. Si analizamos hoy la gran cantidad de aportaciones de educación a distancia en el mundo, observaremos que muchas de ellas no han traspasado aún esta primera generación, siendo en buena parte de los países la forma más popular de desarrollar la educación a distancia, caso específico de México ante el confinamiento del COVID-19. 
La enseñanza multimedia a distancia, o en terminología de Garrison, segunda generación se podría situar a finales de los años sesenta (creación de la Open University Británica), viviendo en nuestros días su probable final. Radio y televisión, medios presentes en la mayoría de los hogares, son las insignias de esta etapa. El texto escrito comienza a estar apoyado por otros recursos audiovisuales (audiocasetes, diapositivas, videocasetes, etc.). El teléfono se incorpora a la mayoría de las acciones en este ámbito, para conectar al tutor con los alumnos. En esta segunda generación, al quedar roto el concepto de clase tradicional, las posibilidades de interacción presencial, son escasas.

Es importante señalar que predomina el concepto de educación a distancia entendido como: proceso educativo en el que toda o la mayor parte de la enseñanza es llevada a cabo por alguien que no comparte el mismo tiempo y/o espacio que el alumno, por lo cual toda o la mayor parte de la comunicación entre profesores y alumnos se desarrolla a través de un medio artificial, sea electrónico o impreso. Son elementos prioritarios el diseño, producción y generación de materiales didácticos, dejando en segundo lugar la interacción con los alumnos y de éstos entre sí, son hechos demostrables de estas dos primeras generaciones de la educación a distancia.

El educando fue un invento del siglo XVI; no existía en los tiempos, digamos, de Shakespeare. Hasta entonces, estaba fundido en el mundo adulto y no había nada que pudiera llamarse infancia en el sentido que nosotros damos a la palabra. El niño de hoy está creciendo absurdamente, porque vive en dos mundos y ninguno de ellos lo impulsa a crecer. Crecer; esta es nuestra nueva tarea, y ella es total. La mera instrucción no basta (Mackluham,1969, p.18).

El mismo Mackluham describe la ausencia de una esctructura didáctica propia para el proceso de enseñanza aprendizaje con el uso de la nueva tecnología, sobre todo hace una crítica sobre el uso de la televisión concentrada en deformar la conciencia del sujeto; y la otra, la escuela sin un planteamiento específico para crear reflexión y conciencia en el educando:

Hay un mundo de diferencia entre el aula y el ambiente de información eléctrica integrada del hogar moderno. Al niño televidente de hoy se lo afina con el diapasón de las noticias "adultas" al minuto: inflación, disturbios, guerra, impuestos, delicuencia, beldades en traje de baño, y queda perplejo cuando ingresa al ambiente del siglo XIX que caracteríza todavía al sistema educacional, con información escasa pero ordenada y estructurada por patrones, temas y programas fragmentados y clasificados. Se trata, naturalmente, de un ambiente muy semejante al de cualquier fábrica con sus invetarios y líneas de montaje (Mackluham, 1969, p. 18).

Durante los años 1969 a 1973 se implementa el uso de la televisión en los sistemas educativos y conjuntamente se inicia el desarrollo de las Tecnologías de Información y Comunicación, lo cual consiste específicamente en la fabricación de equipos de computadora, sistemas para operación de los equipos y su interconexión, pero lo más importante radica en lo referente a los sistemas de información que generan las empresas y organizaciones: productos; servicios; inventarios; datos de clientes; zonas específicas para desplazar productos, bienes y servicios. Todo esto reunidos en una base de datos, que a través de la comunicación: mensajes, notas, carteles, avisos, entre otros que genera en los sujetos necesidades de consumo. 
Este nuevo ambiente se denomina las nuevas Tecnologías de Información y Comunicación y define un nuevo propósito: el uso de las TIC's en los sistemas educacionales, el cual tienen impacto a partir de los años noventa. Provocando resistencia en los docentes y visión corta de las autoridades educativas al no invertir en recursos e insumos.

Por su parte el gran pedagogo Iván Illich promotor de muchas de las reformas educativas en todo el mundo, en su obra "La Sociedad desecolarizada" se adelanta a la crítica y reflexión del nuevo modelo educativo en las escuelas al proponer las "Características generales de las nuevas instituciones educativas formales" las cuales se sustentan en tres objetivos: a) proporcionar a todos aquellos que lo quieren el acceso a recursos disponibles en cualquier momento de sus vidas; b) financiar a todos los que quieran compartir lo que saben, así como generar acciones que permitan encontrar a quienes quieran aprender de ellos; c) dar a todo aquel que quiera presentar al público un tema de debate, la oportunidad de exponer su argumento.

Este sistema educativo debe estar sustentado con garantías constitucionales. Los educandos no deben ser sometidos a un currículum obligatorio, o a una discriminación fundada en la posesión o carencia de un certificado o diploma. Ni obligar tampoco al público a mantener, mediante una retribución regresiva, un gigantesco aparato profesional de educadores y edificios que de hecho disminuye las posibilidades que el público tiene de aprender los servicios que la profesión está dispuesta a ofrecer al mercado. Usar la tecnología moderna para lograr que la libre expresión, la libre reunión y la prensa libre fuesen universales y, por consiguiente, plenamente educativas..... la escuela es una institución construida sobre el axioma de que el aprendizaje es el resultado de la enseñanza. Y la sabiduría institucional continúa aceptando este axioma, pese a las pruebas abrumadoras en sentido contrario". El estado de ánimo de 1971 es propicio para un cambio importante de dirección en busca de un futuro esperanzador (Illich, 1985, p. 44).

Importante reflexión que cuestiona al sistema escolarizado y que convoca a diferentes especialistas en educación a replantear las características de las nuevas instituciones educativas formales, la Organización de las Naciones Unidas para la Educación, la Ciencia y la Cultura (UNESCO) retoma la idea de replantear la función de las escuelas y nombran a una comisión especial liderada por el Ex Ministro de Educación de Francia y presidente de la Comisión Internacional sobre el Desarrollo de la Educación Edgar Faure en febrero de 1971 a mayo de 1972. Los integrantes de dicha comisión fueron: Felipe Herrera (Chile), profesor de Universidad de Chile, Ex-Presidente del Banco Interamericano de Desarrollo y Ex-Director Ejecutivo del Fondo Monetario Internacional; Abdul-Razzak Kaddoura (Siria), agregado en el Laboratorio de Física Nuclear de la Universidad de Oxford y miembro del Consejo de Gobernadores de la Agencia Internacional de la Energía Atómica; Henri López (República Popular del Congo), entonces Ministro de Educación y en ese momento Ministro de Asuntos Exteriores; Arthur V. Petrovski (URSS), Secretario de la Sección de Psicología y Fisiología del Crecimiento, de la Academia de Ciencias Pedagógicas; Majid Rahnema (Irán), Ex-ministro de Enseñanza Superior y Ciencias, y antiguo Vicepresidente del Consejo Económico y Social de las Naciones Unidas; F. Champion Ward (EE. UU.), consejero para la educación internacional en la Fundación Ford, y Ex-Decano de Facultad en la Universidad de Chicago. Esta comisión elaboraron el informe intitulado: Aprender a ser. La Educación del futuro, su crítica se centro en la necesidad de implementar cambios 
en los sistemas educativos en relación con el contexto social, las nuevas tecnologías y la consideración de los aprendizajes informales y no formales que se realizan en otros contextos ajenos a la escuela.

Dondequiera que encontremos un sistema educativo tradicional que ha resistido la prueba del tiempo y generalmente se pensó que no necesitaba más que algunas mejoras menores ocasionales, algunos ajustes más o menos automáticos, actualmente está desatando una avalancha de críticas y sugerencias que a menudo van hasta el punto de cuestionarlo en su totalidad. Algunos jóvenes ahora protestan más o menos abiertamente contra los modelos pedagógicos y tipos de instituciones que se les imponen, aunque no siempre es fácil delimitar la influencia de este fenómeno en particular, con su vago malestar y destellos de rebeldía". Reporte de la Comisión Internacional para el Desarrollo de la Educación. UNESCO, presentado a 130 miembros en otoño de 1972 en Paris. Texto de primera página del Preámbulo que define cuatro postulados centrales (Faure et al., 2013, p. xxi):

1. Existencia de una comunidad internacional...(que) se expresa por la comunidad de aspiraciones, de problemas y de tendencias y por la convergencia hacia un mismo destino.

2. La creencia en la democracia, concebida como el derecho de cada uno de los hombres a realizarse plenamente y a participar en la construcción de su propio porvenir.

3. El desarrollo tiene por objeto el despliegue completo del hombre en toda su riqueza y en la complejidad de sus expresiones y compromisos.

4. La educación, para formar a este hombre completo ... sólo puede ser global y permanente.

La educación se extiende a lo largo de toda la trayectoria de vida del ser humano. Es incorrecto definirla solamente desde la escuela, ni ligarse a la circunstancia del aula. El proceso de educación debe definirse como una red pluridimensional, visualizada en múltiples direcciones, con componentes heterogéneos y cambiantes. El ser humano es un aprendiz en cualquier momento y circunstancia. Muchos de los aprendizajes se han logrado fuera del sistema escolar. Podemos aprender en un viaje, en el trabajo, en el circulo familiar, como miembbros de un grupo social, a través de los medios sociales de comunicación, pero principalmente mediante la reflexión que realiza el propio sujeto. El contexto de una sociedad del aprendizaje se sobrepone al concepto limitativo de la sociedad escolarizada. Ante esta reflexión se resalta que el paso siguiente no es cerrar las escuelas sino reflexionar sobre las limitaciones de la escuela tradicional y transformar sus funciones enfocados en tres dimensiones específicas: las funciones educativas que asumen las instituciones de producción y servicio; la educación informal brindada por grupos más o menos organizados, y el aprendizaje-enseñanza individual sustentado en la motivación y el autodidáctismo.

Al inicio de los años setenta se visualiza al mundo como una aldea global (Mackluham,1969). Pero esta descripción se queda corta si se consideran los innuerables vínculos generados entre las naciones, los cuales dan origen en el campo de la educación a una red transnacional que agrupa a organismos internacionales para elaborar indicadores y estrategias para el diseño de políticas educativas, el análisis de los cambios de ideales y pensamiento de los jóvenes que transitan en forma rápida de una frontera a otra y dan origen a un intercambio 
de ideas, conocimientos, tecnología y valores a escala mundial.

Se generaron; por ejemplo, acuerdos multinacionales, como el Convenio Andrés Bello, el cual concreta las bases para programas regionales de estudios, equivalencia e innovación. La red transnacional permite a las naciones diseñar sus propias redes pluridimensionales. A su vez, éstas pasarían a ser estructura de la primera. Así, se fomentarían la diversidad de los diseños nacionales y la interrelación entre los diferentes países.

\section{Contexto educativo en 2020 y la Pedagogía Virtual}

Las reflexiones de Faure et al. (2013) adquieren relevancia ante los sucesos del confinamiento por el COVID-19. Invitan a reflexionar sobre los futuros de la educación. El cierre total de muchas escuelas en todo el mundo para evitar contagios y propagación del coronavirus han generado, por parte de las autoridades educativas, el diseño de acciones específicas para dar seguimiento a las actividades de los estudiantes en casa, como ejemplo: programas en la televisión; emisión de pláticas por la radio; imprimir cuadernillos de ejercicios para los alumnos; enviar instrucciones por correo electrónico; videoconferencias por parte de los docentes; usar las redes de Facebook, WhatsApp, entre otras, para enviar tareas y trabajos a los alumnos para mantener la continuidad de la enseñanza.

Son muchas y diferentes las acciones que han tomado las autoridades educativas, así mismo; debe reconocerse la creatividad y espontaneidad de muchos docentes. Sin embargo, la contingencia impuesta por el coronavirus puzo en evidencia la deficiencia en infraestrcutura (reporte que publica UNESCO, 2020, p. 114) en los países de América Latina y El Caribe; muchas escuelas aún no cuentan con energía eléctrica, el $40 \%$ de los alumnos de las escuelas de educación básica en México no tienen computadora en casa, ni en la escuela. La decisión emitida por la autoridad educativa de iniciar en septiembre clases por televisión, no define la forma en que los docentes realizarán su intervención con los estudiantes. Algunos supervisores, directores y docentes decidieron, por cuenta propia, contratar los servicios de Google Classrom, pero se carece de una articulación de los procesos pedagógicos para diseñar clases en las aulas virtuales.

Debemos analizar los resultados de las decisiones apresuradas que tuvieron que tomarse durante la pandemia para hacer frente a la continuidad de los servicios educativos y que han ocasinado el uso improvisado de las herramientas digitales: plataformas, aplicaciones para videoconferencias y otros materiales del internet, es importante señalar que la intervención docente ha sido de forma desorganizada y sin una fundamentación pedagógica clara. Tal situación nos motiva para señalar y resaltar los conceptos didácticos y pedagógicos que deben ser considerados en el uso de las herramientas digitales y permitan planificar las clases que se imparten en línea.

\section{Conceptualización de la Educación en Línea}

El concepto de educación en línea surge del término e-learning, de la misma manera que los términos: e-mail, e-comerce, e-business. Deriva de la expresión "electronic learning" su traducción del inglés es: "aprendizaje electrónico" y su aplicación a la educación tiene sustento en el campo específico de las tecnologías de la información y la comunicación, que incorporan el uso del internet a los sistemas de gestión del aprendizaje e incorporan diferentes multimedios para la enseñanza, como una nueva estrategia para lograr el aprendizaje. 
Las tecnologías de información y de comunicación, se refieren a la gran variedad y cantidad de herramientas que nacieron a partir del Web Course Tools primer LMS, que da origen a una nueva forma de organizar los procesos de gestión del aprendizaje de una empresa, institución o escuela. Su implementación surge por el uso de internet, y los distintos formatos de presentación de la información: páginas web, imágenes, audio, video, elementos interactivos (todos estos en formatos digitales), que han permitido el desarrollo de nuevas estrategias y materiales didácticos que aportan elementos para lograr un sistema eficaz de enseñanza y aprendizaje. Por otra parte también deben considerarse las distintas herramientas de comunicación: chat, e-mail, foros, wikis, blogs, mensajería, teléfono IP, teleconferencia y las que aún siguen apareciendo y están en vías de desarrollo (televisión IP, por ejemplo).

Las herramientas digitales y el uso del internet están dirigidas hacia el aprendizaje, tanto de la educación presencial como de la educación a distancia; considerando que deben incluir una estrategia de enseñanza clara, precisa, coherente y planificada. Sus principios y fundamentos deben ajustarse a las teorías pedagógicas de enseñanza y aprendizaje, considerando que los sistemas de gestión del aprendizaje, nombrados comunmente como "plataformas tecnológicas" integran acciones indispensable para la aplicación e implementación de contenidos, ejercicios, actividades y evaluaciones de los procesos de enseñanza y aprendizaje. La educación en línea ha sido un término controvertido, por un lado, porque tiende a poner a la tecnología en el centro y por otro lado, porque plantea una nueva conceptualización sobre el aprendizaje, es decir una estrategia diferente de enseñanza para lograr el aprendizaje, a esta nueva estrategia la definiremos como Pedagogía Virtual.

Así como la aparición de los libros generó la definición de un nuevo modelo pedagógico; hoy las computadoras, tabletas y teléfonos moviles deben construir su propio modelo pedagógico, los libros y el hardware son las herramientas que se usan como instrumentos en el proceso educativo. El aprendizaje y la enseñanza seguirán definiéndose, esencialmente, como actividades humanas.

La evolución del software de las computadoras, sus esquemas y combinaciones con los medios de comunicación, integrados en el internet. Han generado una fuerte presencia en varios entornos de la vida cotidiana, no solamente laboral, científica o en la producción. Actualmente hay aplicaciones de software para diferentes entornos y actividades, la educación y la pedagogía no son la excepción; aunado a esto los medios de comunicación que han surgido bajo los estándares del internet han hecho que la forma de vivir y actuar se esten transformando. De tal forma que se pueden considerar como herramientas necesarias para la educación y para el aprendizaje.

\section{Definición de Pedagogía Virtual en el nuevo contexto de la educación en línea}

Hablar de pedagogía virtual lo hacemos refiriendonos al concepto de modelo pedagógico, ya que constituyen una interpretación de la concepción del mundo y de las ideologías que enmarcan la vida intelectual y la generación de saberes filosóficos y científicos en cada época y en cada sociedad históricamente establecida. El modelo es un instrumento analítico para describir, organizar e interpretar las diferencias que han preocupado al hombre desde siempre en los procesos educativos. Los aspectos esenciales que debemos tener en cuenta y que debemos respondernos son: 
- ¿Qué tipo de hombre nos interesa formar?

- ¿Con qué estrategias técnicos-metodológicas?

- ¿A través de qué propósitos y contenidos?

- ¿Qué debe evolucionar en el proceso de formación?

- ¿Quién predomina o dirige el proceso, si el alumno o el maestro?

Estos aspectos escenciales forman categorias o aspectos que constituyen variables que se articulan e interrelacionan en cada construcción teórico pedagógica, dando origen a múltiples combinaciones dinámicas que conforman los modelos pedagógicos los cuales se caracterizan por definir los siguientes elementos: meta; relación profesor-alumno; métodos educativos y de enseñanza; prósitos-contenidos y desarrollo individual y social (Flórez Ochoa, 1994, p. 175).

Los modelos no son esquemas rígidos ni estructuras estáticas divididas. Deben ser asimiladas como sistemas abiertos, de flujos e interacción interna y permanente, cuya intensidad y dirección siempre habría que determinar en cada discurso pedagógico específico y cuyo funcionamiento, en la "praxis" educativa, habría de precisarse cada vez bajo las condiciones histórico sociales en el contexto presente y desde el análisis de la pedagogía de la cotidianeidad.

El Modelo Pedagógico Cognitivo que evoluciona de los modelos tradicional y conductista, ha sido definido como desarrollista o neoliberal, definimos sus estructura con los siguiente elementos. (Flórez Ochoa, 1994, p.188)

Meta: acceso al nivel superior de desarrollo intelectual según las condiciones biosociales de cada sujeto.

Relación profesor-alumno: donde el docente es es facilitador-estimulador de experiencias.

Método: basado en la creación de ambientes y experiencias de afianzamiento según cada etapa, el niño es un "investigador".

Contenidos: basado en experiencias que faciliten acceso a estructuras superiores de desarrollo, el niño construye sus propios contenidos de aprendizaje.

Desarrollo: progresivo, secuencial a estructuras mentales cualitativa y jerárquicamente diferenciada.

Continuando con la misma propuesta conceptual de Flórez Ochoa (1994), proponemos la definición del Modelo Pedagógico Virtual sustentados en la iniciativa de UNESCO (2020) que convoca a sumar ideas para definir los futuros de la educación, se describe de la siguiente manera:

Meta: aprender a convertirse para transformar el futuro de la humanidad y del planeta.

Relación profesor-alumno: ambos sujetos que tratan de suscitar diálogos sobre el conocimiento y el aprendizaje. 
Método: autonomía e independencia, rasgo ciudadano de toda sociedad que pretende un enfoque humanista en su proyecto educativo, para asumir responsablemente una actitud consciente y de libertad ante los actuales problemas de nuestro entorno.

Propósitos-Contenidos: aprendizajes clave y conocimiento a la luz de los enormes desafíos y oportunidades.

Desarrollo: promover oportunidades de aprendizaje para todos durante toda la vida.

Tomando en cuenta que el principal elemento que hace viable la creación de un modelo pedagógico es el currículum, del cual se construyen y acuerdan los diferentes planes de estudio en correspondencia a cada uno de los diferentes niveles educativos. El currículum es definido como instrumento que contiene los resultados que se esperan de la educación y los procesos formativos que se definen para los estudiantes; la especificación de los recursos que permiten alcanzar los propósitos educativos; definición de los fundamentos del acto educativo; detrminación de los procesos de evaluación y principalmente los programas de estudio con sus contenidos y actividades de aprendizaje.

Estos elementos curriculares prescriben, orientan, fundamentan y norman todo el proceso de enseñanza y aprendizaje, pero debemos reconocer la parte esencial que da viabilidad al proyecto curricular y este elementos corresponde a la intervención del docente y se materializa con la planificación didáctica. Con implementación del proyecto curricular nos referimos al proceso de ejecución, desarrollo, concreción, operación o puesta en práctica de aquello que se propone, plantea o se determina en el diseño del currículo. Para Ibarrola (2012) la noción de currículo no puede limitarse sólo a la idea de objetivos en un documento o en una serie de documentos prescriptivos, orientadores y articuladores de la institución escolar; es necesario definirlo desde la intervención del docente, con lo que sucede con él en su puesta en práctica en el entramado de las herramientas e infraestructura de la institución escolar. La intervención docente, ante el confinamiento por la pandemia, se ha visto minimizada y las acciones espontáneas que el cuerpo docente han asumido en forma personal han generado desfases en los procesos pedagógicos. La manera en que se ha tratado de realizar, por parte del docente, su aproximación a los procesos de enseñanza en lo que podemos llamar el aula virtual, se ha realizado sin una adecuada estrategia en el uso de las herramientas digitales, por ejemplo pensar que la herramienta zoom diseñada para uso de videoconferencias; el envío de mensajes por Facebook o WhatsApp cubren en forma integral los procesos del aprendizaje, sería un error aceptar este postulado, ya que nos remite a la conceptualización de la enseñanza del proceso acotado por el modelo pedagógico tradicional, que solamente se basa en la transmisión frontal del conocimiento, sin generar la participación activa del alumno, y sin involucrarlo en procesos que le permitan la construcción del conocimiento. 
Figura 1. Referentes de la Pedagogía Virtual. Elaboración propia

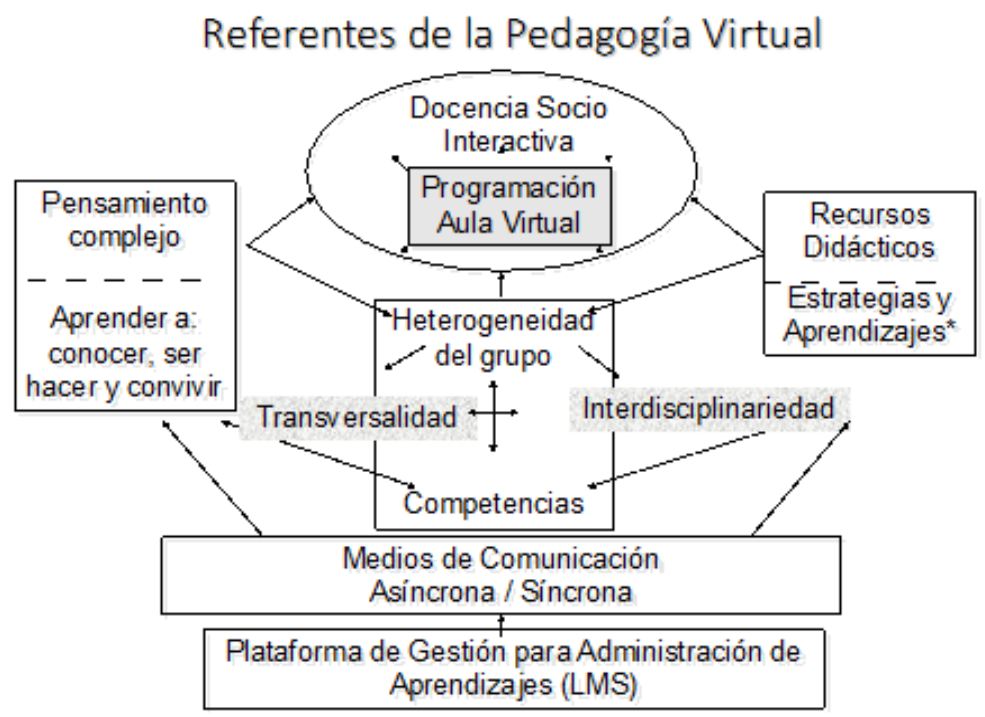

* Estrategias: Combinación de una serie de procedimientos (trabajo del profesor, acciones del alumno, manejo de materiales y recursos, selección de bibliografía, organización del grupo, etc.) que se organizan en actividades para lograr los aprendizajes.

La implementación curricular con el uso de herramientas tecnológicas debe considerar las características y condiciones del docente que puedan facilitar la puesta en práctica de lo propuesto en el diseño: perfil docente, formación en el uso de de herramientas de comunicación digital, diseño de actividades en las plataformas de gestión para la administración del aprendizaje, el uso correcto de las videoconferencias y su pensamiento al respecto sobre los planteamientos educativos, entre otros elementos; pero también debemos hacer referencia a las características de los alumnos que pueden llegar a facilitar o impedir lo propuesto en el proyecto curricular y contribuir a que logren los aprendizajes que se proponen. Entre las características que puedan definirse, la principal deberá ser: que los docentes y los alumnos tengan acceso a internet, que tengan los equipos necesarios para conectarse, que tengan un espacio propio y adecuado para permanecer sentado durante varias horas para realizar las actividades de aprendizaje que se proponen en la implemetación del currículum.

Deben ser considerados los intereses y expectativas de los alumnos o la percepción que tienen sobre su papel en el proceso educativo realizado con el uso del internet y las herramientas digitales. Así, pues, la implementación curricular debe centrarse en la práctica educativa que se configura en el contexto escolar denominado aula virtual, la cual se caracteriza por la comunicación sincrona y asíncrona, las interacciones y los procesos que se establecen entre los diferentes usuarios de las redes sociales, los contenidos y métodos educativos de la pedagogía virtual.

El aula virtual, no es solamente un mensaje en redes sociales o una brillante videoconferencia, debe ser un espacio integrador que incluye, textos, mensajes, libros, gráficos descriptivos, ejercicios para el aprendizaje, lugares específicos para la interacción y conver- 
sación por parte de los alumnos para completar sus ideas y descubrir nuevos contenidos que promuevan su aprendizaje. El aula virtual debe ser un espacio en el cual confluyen todos los alumnos, todas las ideas y todos los materiales que posibiliten el aprendizaje.

La implementación del curriculum, en el aula virtual, debe sustentarse en la conceptualización de docencia, entendida como actividad profesional transformada, como toda práctica social y de forma significativa, con el impacto de las condiciones propias del desarrollo de las tecnologías de informática y comunicación, considerando que ha transitado desde una dinámica fluctuante entre una práctica empírica y asilada, que se perfecciona con la experiencia, hasta ser considerada como práctica íntimamente articulada a procesos intelectuales, sustentados en la formación didáctico pedagógica y principalmente en la socialización de la docencia.

En el contexto de las instituciones educativas, la docencia se ha diversificado por el cumplimiento de tareas académicas cada vez más complejas, que requieren de una formación específica de los profesores no sólo para el dominio de nuevas estrategias didácticas, sino también para la apropiación de teorías y métodos de investigación que les permitan avanzar paralelamente en los aspectos pedagógicos y disciplinarios, así como en el uso de las herramientas digitales en los procesos de enseñanza y aprendizaje.

Por su parte, el docente se encuentra frente a serios compromisos que implican su participación en las recientes exigencias planteadas por las estrategias políticas de incorporación a los estándares internacionales, las cuales le exigen: dominio de las técnicas de trabajo grupal; conocimiento para la planeación del trabajo en aula, así como el manejo de estrategias para lograr el aprendizaje de los estudiantes haciendo uso de las herramientas digitales.

Como ejercicio profesional de la educación, con el uso de las herramientas digitales la docencia se convierte en una práctica sustentada en la planeación reflexiva y propositiva de la enseñanza para lograr el diseño e implementación de estrategias para el aprendizaje, actividad que debe ser realizada por el conjunto de docentes; este trabajo de reflexión y proposiciones en el colectivo de los profesores se denomina docencia, y su acción principal consiste en ser el articulador entre la teoría y la praxis social.

El trabajo del docente en el modelo presencial se ha caracterizado por ser individualista, las decisiones concernientes a su práctica son de índole unipersonal, principian y terminan en cada aula, taller o laboratorio. En los sistemas de enseñanza virtual y con uso de las herramientas digitales el docente se propone avanzar hacia la conceptualización del trabajo grupal, interdisciplinario y colegiado, para transformarse en docencia socio interactiva, la cual va más allá de las actividades de índole unipersonal y la simple transmisión de conocimientos. Es una conceptualización, en vías de reconocimiento, con una actividad compleja que requiere para su ejercicio la comprensión del fenómeno educativo (Renteria, 2015, pp. 217-218).

Por tanto definiremos a la docencia socio interactiva de las instituciones educativas en el proceso de educación en línea, puesto que se trata de un planteamiento que busca alcances institucionales, donde, para lograrlo, se requiere no solamente de la participación convencida de la planta docente o cuerpo académico, sino también, y en primer lugar, de la voluntad política de las autoridades gubernamentales y administrativas de cada comunidad geográ- 
fica, sustentándose en una sólida visión del trabajo de conjunto, y sustentado por dos ejes: evaluación y procesos de mejoras continuas.

El ser para trascender es el compromiso asumido por este grupo, definido como: docencia socio interactiva, integrada por la comunidad académica, la cual se basa en un ideal común y se dirige a objetivos científicos, técnicos y culturales, generando la participación de sus miembros, que se conocen y respetan recíprocamente. Esta comunidad académica está formada por las autoridades educativas, los docentes y los estudiantes, integrados en un modelo pedagógico virtual e interactuando en el aula virtual.

Es de la mayor urgencia que nuestras instituciones educacionales adviertan que ahora se libra una guerra civil entre esos ambientes, creados por medios distintos de la palabra impresa. El aula lleva a cabo una lucha vital por la supervivencia con el mundo "exterior", enormemente penetrante, creado por los nuevos medios informativos. La educación debe apartarse de la instrucción, dejar sus clisés e ir hacia el descubrimiento, hacia el sondeo, la exploración y el reconocimineto del lenguaje de las formas. Hoy, los jóvenes rechazan las metas. Quieren desempeñar roles...R-O-L-E-S. Es decir, compromiso total. No quieren objetivos o puestos fragmentarios, especializados (Mackluham, 1969, p.100).

El acto docente implica siempre tener presente al estudiante que aprende, dado que la enseñanza es una actividad de transmisión frontal, instrumental, transitiva e interactiva, que produce un efecto en quien aprende, por medio de la relación dinámica, interpersonal e intencional que se da entre docente y alumno, a propósito de un contenido científico, tecnológico, cultural y la responsabilidad de trasformar nuestro entorno.

\section{CONCLUSIONES}

El inicio de la educación a distancia adolecía tanto de un espacio permanente para la comunicación e interacción, como del logro de los aprendizajes con mayor asertividad. La aparición del internet ha propiciado ese espacio que permite una comunicación permanente, así mismo facilita la incorporación de un número importante de recursos didácticos: bibliotecas, hemerotecas, videoconferencias y otros tantos; que son identificados por los docentes e in- corporados a un espacio, el aula virtual, la cual hace posible clasificar contenidos, determinar competencias y habilidades a desarrollar, y principalmente permite al docente el diseño de estrategias que faciliten a los estudiantes interactuar con el objeto de estudio y que, de acuerdo al tiempo y ritmo de avance en lecturas y realización de ejercicios, logren apropiarse de dicho conocimiento y desarrollen sus competencias.

Ante el escenario actual, resulta necesario formar a los docentes en el uso de las herramientas digitales con un enfoque pedagógico, para evitar repetir los sucesos de los años setenta en donde se construyeron diversas instituciones de educación y se improvisó una planta docente para dar atención inmediata a los estudiantes matriculados en todo el país, sin antes divulgar el proyecto curricular con los docentes, definir los objetivos de la institución, replantear los objetivos de las asignaturas y sobre todo sin definir el modelo pedagógico que sustenta dicho proyecto curricular; y trajo consigo una población de profesores sin formación pedagógica que les posibilitara realizar la función de docencia. 
Hoy para incorporar a los docentes a la educación en línea y al uso de las nuevas tecnologías, deben ser formados no solamente en el uso de las herramientas digitales, sino también en los fundamentos didáctico pedagógicos que permitan el uso y diseño asertivo de estrategias que faciliten el desarrollo de competencias en el estudiante y principalmente el logro de aprendizajes que permitan transforma a la humanidad y al planeta. La pedagogía virtual debe centrase en el aprendizaje por un motivo muy simple: el crecimiento, el desarrollo y la reducción de la pobreza dependen de los conocimientos y las capacidades que adquieren las personas, no de la cantidad de años que pasan en un aula.

\section{REFERENCIAS}

De Ibarrola, M. (2012). Experiencias y reflexiones sobre el diseño y la evaluación curricular. En: Landesmann, M. (coord.). El currículum en la globalización. A tres décadas de "el currículum pensado y el currículum vivido" (pp. 93-118). México: UNAMFES Iztacala.

Faure, E., Herrera, F., Kaddoura, A., López, H., Petrovski, A. R., Majid. W., \& Frederick C. (2013). Comisión Internacional de Desarrollo de la Educación. UNESCO.

Flórez Ochoa, R. (1994). Hacia una pedagogía del conocimiento. McGraw-Hill.

García Aretio, L. (1987). Eficacia de la UNED en Extremadura. Badajoz: UNED-Mérida.

García Aretio, L. (1991). Historia de la Educación a Distancia. Universidad Nacional de Educación a Distancia (UNED).

Illich, I. (1985). La Sociedad desescolarizada. Edit. Joaquín Mortiz.

Renteria Castro, E. (2015). Educación a Distancia, el uso de nuevas herramientas tecnológicas y la didáctica digital en la docencia universitaria. Diálogos. Las TIC en la Universidad. Comunidad académica comprometida con el desarrollo humano de la sociedad. Universidad Autónoma Metropolitana. http://www.red-inedat.org/dialogos-las-tic-en-la-universidad/

UNESCO (2020). América Latina y El Caribe. Inclusión y educación: Todas y todos sin excepción (1 ed.). Informe de seguimiento de la Educación en el Mundo.

UNESCO. (1972). Reporte de la Comisión Internacional para el Desarrollo de la Educación. Faure Edgar. Herrera, Felipe. Kaddoura, Abdul Razzak. Lopes, Henri. Petrovski, Arthur V. Rahnema, Majid. Ward, Frederick Champion. Recopilación en libro 313p. París, 2013.

\section{BIBLIOGRAFÍA COMPLEMENTARIA}

Brown, A., Sally, G. Á. (2007). Evaluar en la universidad: problemas y nuevos enfoques. Ediciones Narcea.

Díaz Alcaraz, F. (2002). Didáctica y currículo: un enfoque constructivista. Colección Humanidades - Universidad de Castilla-La Mancha.

Gagné, R. M. (1975). Principios básicos del aprendizaje para la instrucción. Diana. 
Gimeno, J. (1992). Investigación e innovación sobre la gestión pedagógica de los equipos de profesores. En La gestión pedagógica de la escuela. UNESCO-OREALC. Santiago de Chile.

Gimeno, J. (1995). El currículum: una reflexión sobre la práctica. Morata.

Ginoris, O. (2009). Fundamentos didácticos de la educación superior cubana. Selección de lecturas. Editorial Félix Varela.

Glazman, R. (2005). Las caras de la evaluación educativa. UNAM.

Knight, P. (2006). El profesorado de educación superior: formación para la excelencia (2a ed.). Narcea ediciones.

Norton, Lin; Richardson, John; Hartley, John (2005). Teachers 'beliefs and intentions concerning teaching in higher education. Higher Education, 50(4), 537-571.

Pozo, J. I., Scheuer, N., Mateos, M., \& Pérez, M. (2006). Las teorías implícitas sobre el aprendizaje y la enseñanza. En Pozo, Juan; Scheuer, Nora; Pérez, María; Martín, Elena (eds.). Nuevas formas de pensar la enseñanza y el aprendizaje. Las concepciones de Profesores y alumnos. Graó. 